\title{
Anisotropic diffusion for noise removal of band pass signals
}

\author{
Sasan Mahmoodi* \\ School of Electronics and Computer Science, Building 1, Southampton University, Southampton SO17 1BJ, UK
}

A R T I C L E I N F O

\section{Article history:}

Received 10 September 2010

Received in revised form

10 November 2010

Accepted 21 December 2010

Available online 8 January 2011

Keywords:

Noise removal

Band pass signals

Perona-Malik algorithm

Anisotropic diffusion

\begin{abstract}
A B S T R A C T
A noise removal method for band pass signals based on the anisotropic diffusion algorithm originally put forward by Perona and Malik is proposed in this paper. The anisotropic smoothing algorithm proposed here is for band pass signals modulated with a constant carrier frequency. A partial differential equation to smooth band pass noisy signals is derived. The propagator of this differential equation is also analytically calculated in this paper. An appropriate linear operator is then considered here for such band pass signals to form an anisotropic diffusion algorithm. The algorithm proposed here demonstrates better performance for band pass noisy signals containing discontinuities in comparison with the traditional Perona-Malik (PM) algorithm and is robust in the presence of excessive noise with SNR less than unity.
\end{abstract}

(c) 2011 Elsevier B.V. All rights reserved.

\section{Introduction}

Noise removal for signals and images has received considerable attention in recent years. A nonlinear noise removal algorithm of low pass signals and images containing discontinuities is proposed in the pioneering work of Perona and Malik (PM) [1] based on anisotropic diffusion. Perona and Malik claim that their anisotropic diffusion equation has no additional maxima (minima) that do not belong to the original image. This claim is challenged in Refs. [2,3]. Excessive noise may produce a gradient comparable to image edges and features. In such cases, PM algorithm may therefore fail $[2,4-6]$. A regularization procedure can however improve the performance of the PM algorithm in the presence of excessive noise. An edge enhancing functional is also proposed in Ref. [7] for direct edge enhancement to treat low contrast and edge blurred images. A generalization for $3 \mathrm{D}$ volumetric images is proposed in Ref. [8]. An extension for vector valued images such as color images is also proposed in Ref. [9]. Black et al. [10] propose a robust estimation procedure to estimate a piecewise smooth image from a noisy input image and demonstrate improved automatic stopping of diffusion process with the

\footnotetext{
*Tel.: +44 238059 2075; fax: +44 2380594498 .

E-mail address: sm3@ecs.soton.ac.uk
}

preservation of sharp boundaries and better continuity of edges compared to the PM algorithm. A fast anisotropic smoothing method using curvature-preserving PDEs is proposed in Ref. [11] to denoise multi-valued images. A selective method for image smoothing is also proposed in Ref. [12] by employing an edge-strength function, which improves the quality of the preservations of image details. The contribution of this paper is to propose a nonlinear algorithm for noise removal of band pass signals containing discontinuities. This work is therefore considered to be an extension of the PM algorithm for signals modulated with a sinusoidal signal (carrier) with a constant frequency. The paper is structured as follows. In Section 2, a mathematical argument is presented to generalize the notion of diffusion equation for band pass signals. Section 3 deals with the generalization of the nonlinear anisotropic diffusion originally proposed in Ref. [1] for band pass signals and its implementation issues. Numerical Results are presented in Section 4 and finally the paper concludes in Section 5.

\section{Mathematical framework}

\subsection{Statement of problem}

The anisotropic diffusion algorithm proposed in Ref. [1] is based on the solution of the diffusion (heat) equation when the diffusion coefficient is not constant over the 
space. It is well known that the propagator of linear heat equation is a Gaussian function whose variance is proportional to (virtual) time associated with the (evolving) smoothed signal. Such a propagator has a smoothing property, which removes the noise from the original noisy signal. However this propagator also smoothes discontinuities in the original signal. In order to preserve discontinuities of the original signal and simultaneously remove the noise, the PM algorithm [1] sets the diffusion coefficient to zero where there are discontinuities in the original signal. The discontinuities are detected by examining the gradient of the evolving signal. In this paper, a band pass signal (a piecewise continuous low pass signal modulated with a high frequency carrier with constant frequency) contaminated with additive Gaussian noise is considered for noise removal and smoothing. It is important to note that the original PM algorithm removes the high frequency component (carrier) of the original band pass signal, since it behaves like a low pass filter. Therefore a new method is required to remove the noise and also to preserve discontinuities and high frequency carrier.

\subsection{Heat equation for band pass signals and its propagator}

Let us consider the one dimensional heat equation for the smoothing of low pass signals as follows:

$\frac{\partial u}{\partial t}=K \frac{\partial^{2} u}{\partial x^{2}}$

with initial condition

$u(x, 0)=y(x)$

where $u: R^{2} \rightarrow R, y: R \rightarrow R$ and $K>0$ are the smoothed low pass signal at the evolution (virtual) time $t$, the

a

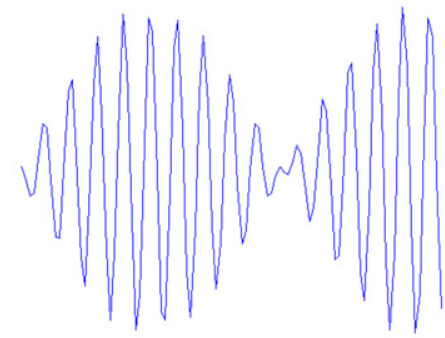

C

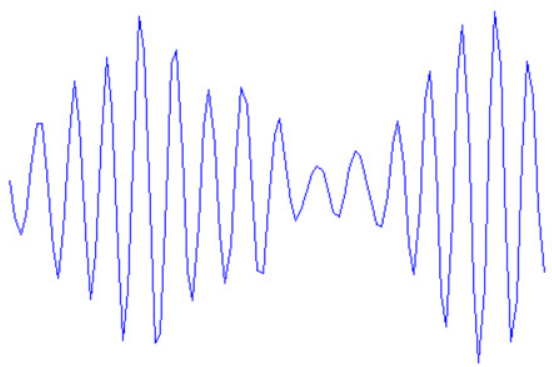

original low pass noisy signal and a constant respectively. It is well known that the propagator of Eq. (1) is written as

$P_{L}(x, t)=\frac{1}{\sqrt{\pi K t}} \exp \left[-\frac{x^{2}}{4 K t}\right]$

It is easy to see from Eq. (3) that propagator (3) is a low pass Gaussian filter removing high frequency components.

Let us now derive a heat equation preserving a specific frequency $\omega_{0}$ and filtering any other frequency. Let us denote $h(x, t): R^{2} \rightarrow C$ as a complex valued band pass signal at virtual time $t$ represented as

$h(x, t)=u(x, t) \mathrm{e}^{i \omega_{0} x}$

where $u(x, t)$ and $\omega_{0}$ are a low pass signal at virtual time $t$ satisfying Eq. (1) and carrier frequency, respectively $(i=\sqrt{-1})$.

By solving Eq. (4) for $u$ and replacing it in Eq. (1), we arrive at the following differential equation:

$\frac{\partial h}{\partial t}=K\left(\frac{\partial^{2} h}{\partial x^{2}}-2 i \omega_{0} \frac{\partial h}{\partial x}-\omega_{0}^{2} h\right)$

with initial condition

$h(x, 0)=y(x) \mathrm{e}^{i \omega_{0} x}$

Eq. (5) with initial condition (6) is a heat equation preserving the carrier with frequency $\omega_{0}$. The propagator of Eq. (5) is derived in the following theorem:

Theorem 1. The propagator of Eq. (5) is a Gabor type complex valued function derived as

$P(x, t)=\frac{\exp \left(-\left(x^{2} / 4 K t\right)+i \omega_{0} x\right)}{\sqrt{\pi K t}}$

The proof is presented in Appendix A.

b

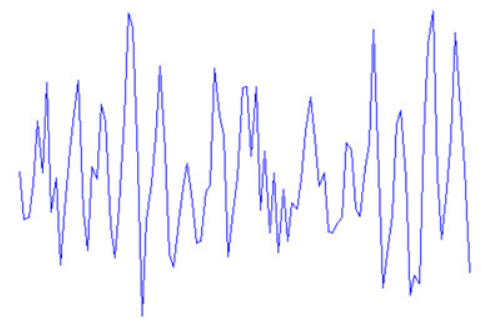

d

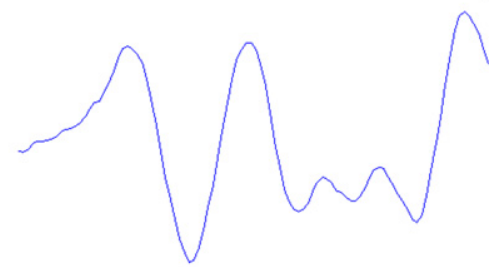

Fig. 1. (a) Original noiseless signal with carrier frequency $\omega_{0}=1$. (b) Noisy signal contaminated with additive Gaussian noise with SNR=1.64. (c) Smoothed signal obtained by convolving noisy signal with propagator (7) with $\omega_{0}=1, K=1, t=20$, and filter size $=11$. (d) Smoothed signal obtained by convolving noisy signal with propagator (3) with $K=1, t=20$, and filter size $=11$. 
At this stage, it is interesting to compare numerically the smoothing effects of propagators (3) and (7) on a band pass signal with carrier frequency $\omega_{0}$ when there is no discontinuity in the original signal (linear case). To this end, the solutions of Eqs. (5) and (1) are calculated by convolving propagators (7) and (3) respectively, with the original signal as an initial condition. Fig. 1(a) depicts an original noiseless signal with a carrier frequency $\omega_{0}=1 \mathrm{rad} / \mathrm{s}$. Additive Gaussian noise is added to the signal shown in Fig. 1(a) to obtain noisy signal shown in Fig. 1(b) with SNR=1.64. Propagators (7) and (3) are convolved with the noisy signal shown in Fig. 1(b) to obtain the smoothed signals shown in Fig. 1(c) and (d), respectively. It is clear from Fig. 1(c) and (d) that propagator (7) preserves the high frequency structure of the original noiseless signal while removing the noise; however propagator (3) smoothes both the noise and the high frequency carrier of the original signal. The smoothed signal shown in Fig. 1(d) is completely distorted. It is important to notice that the signal considered in Fig. 1 is real valued. In the above experiments, we therefore convolve the original signal with the real part of propagator (7).

\section{Anisotropic diffusion algorithm for band pass signals and implementation issues}

In this section, we consider the problem of noise removal from band pass signals containing discontinuities. We adapt the nonlinear PM method proposed in Ref. [1] for band pass signals. To this end, Eq. (5) is rewritten as

$\frac{\partial h}{\partial t}=K\left(\frac{\partial}{\partial x}-i \omega_{0}\right)^{2} h$

The difference between Eqs. (1) and (8) is that the operator $\partial / \partial x$ has changed to $(\partial / \partial x)-i \omega_{0}$. In the original anisotropic diffusion algorithm (where $K$ is not constant with respect to $x$ ), Eq. (1) is written as

$\frac{\partial u}{\partial t}=\frac{\partial}{\partial x}\left(K \frac{\partial u}{\partial x}\right)$

The PM algorithm proposed in Ref. [1] is based on Eq. (9) by setting $K$ as a function of $x$ so that $K$ is set to zero when there is a discontinuity in the original noiseless signal. According to the PM algorithm, Eq. (9) is implemented in a discrete domain as

$u(t+\Delta t)=u(t)+\Delta t\left(K_{L} D\left(u_{L}\right)+K_{R} D\left(u_{R}\right)\right)$

where $D\left(u_{R}\right)=u(j+1)-u(j), D\left(u_{L}\right)=u(j-1)-u(j), K_{L}=g(\mid u(j-1)$ $-u(j) \mid)$ and $K_{R}=g(|u(j+1)-u(j)|)$. Function $g(y)$ is usually set to one of the following functions:

$g(y)=\exp \left(-\frac{y^{2}}{q^{2}}\right)$

or

$g(y)=\frac{1}{1+\left(y^{2} / q^{2}\right)}$

where $q$ is a parameter set by users.
For band pass signals using Eq. (8), anisotropic diffusion can therefore be written as

$\frac{\partial h}{\partial t}=\left(\frac{\partial}{\partial x}-i \omega_{0}\right)\left(K\left(\frac{\partial}{\partial x}-i \omega_{0}\right)\right) \mathrm{h}$

with initial condition

$h(x, 0)=s(x)$

Before the discrete implementation of Eq. (13) is discussed, we need to understand more about the operator $\partial / \partial x-i \omega_{0}$.

The impulse response of the operator $\partial / \partial x$ is the Heaviside function, i.e. the solution of the following differential equation is a Heaviside function:

$\frac{\partial u}{\partial x}=\delta(x)$

where $\delta(x)$ is the Dirac delta function. Eq. (14) indicates that in discontinuities of a signal, the operator $\partial / \partial x$ produces a maximum represented by $\delta(0)$ in Eq. (14). The rationale of the PM algorithm is that if there are discontinuities in low pass signals, according to Eq. (14) the term $\partial u / \partial x$ in Eq. (9) produces a maximum at such discontinuities.

a

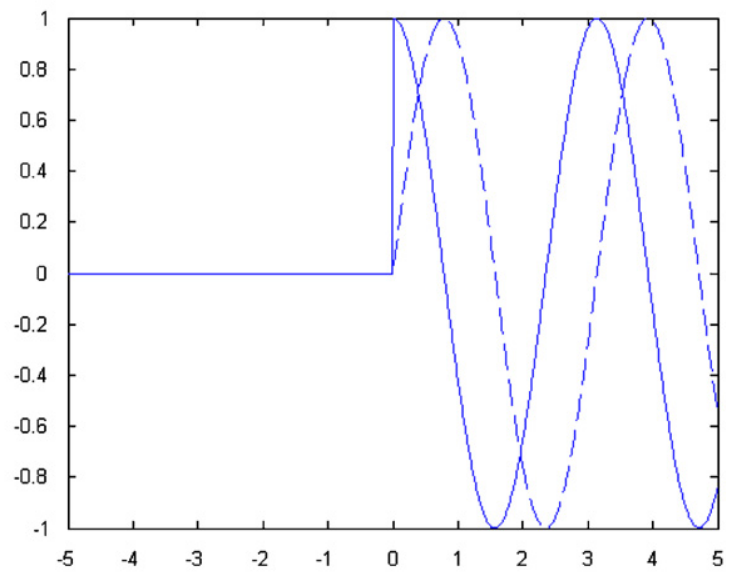

b

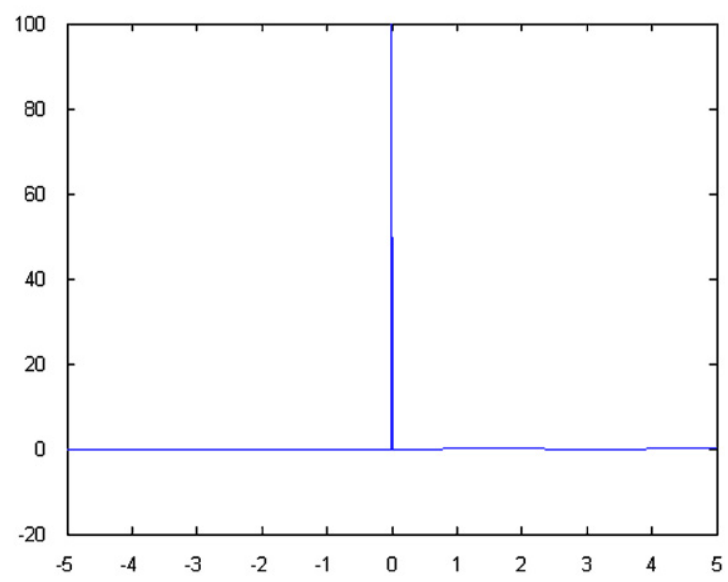

Fig. 2. (a) Complex valued signal (real and imaginary parts in solid and dashed lines) $\omega_{0}=2 \mathrm{rad} / \mathrm{s}$. (b) Numerical evaluation of term $(\partial h / \partial x)-i \omega_{0} h$, where $h$ is complex signal shown in (a) and $\Delta x=0.01$. 
Therefore $K$ should be set to zero anywhere there is a discontinuity to preserve discontinuities of such signals. Similarly the impulse response of $\partial / \partial x-i \omega_{0}$ is $H(x) \mathrm{e}^{i \omega_{0} x}$, i.e. $H(x) \mathrm{e}^{i \omega_{0} x}$ is a solution of Eq. (15) $(H(x)$ is the Heaviside function)

$\frac{\partial h}{\partial x}-i \omega_{0} h=\delta(x)$

Fig. 2(a) shows $h(x)=H(x) \mathrm{e}^{i \omega_{0} x}$ so that the real and imaginary parts are drawn in solid and dashed lines, respectively. Fig. 2(b) depicts $\partial h / \partial x-i \omega_{0} h$ demonstrating a Dirac delta function at $x=0$. In this numerical experiment, we have set $\omega_{0}=2 \mathrm{rad} / \mathrm{s}$ and $\Delta x=0.01$. We notice that as $\Delta x \rightarrow 0$, the term $\Delta h / \Delta x-i \omega_{0} h$ approaches $\delta(x)$. The rationale of our algorithm similar to the PM algorithm is that if there are discontinuities in band pass signals with a constant frequency $\omega_{0}$; the term $\left|\partial h / \partial x-i \omega_{0} h\right|$ in Eq. (13) produces maxima at such discontinuities. Therefore $K$ in Eq. (13) should be set to zero to preserve discontinuities of such band pass signals.

A discrete implementation of Eq. (13) is written as

$h\left(t_{k}+\Delta t\right)=h\left(t_{k}\right)+\Delta t\left(K_{L} D_{\omega}\left(h_{L}\right)+K_{R} D_{\omega}\left(h_{R}\right)-i \omega_{0} K_{R} D_{\omega}\left(h_{R}\right)\right)$

where

$D_{\omega}\left(h_{R}\right)=\frac{h\left(x_{j}+\Delta x\right)-h\left(x_{j}\right)}{\Delta x}-i \omega_{0} h\left(x_{j}+\Delta x\right)$,

$D_{\omega}\left(h_{L}\right)=\frac{h\left(x_{j}-\Delta x\right)-h\left(x_{j}\right)}{\Delta x}+i \omega_{0} h\left(x_{j}\right)$,

$K_{R}=\frac{g\left(\left|\frac{h\left(x_{j}+\Delta x\right)-h\left(x_{j}\right)}{\Delta x}-i \omega_{0} h\left(x_{j}+\Delta x\right)\right|\right)}{\Delta x}$, and

$K_{L}=\frac{g\left(\left|\frac{h\left(x_{j}-\Delta x\right)-h\left(x_{j}\right)}{\Delta x}+i \omega_{0} h\left(x_{j}\right)\right|\right)}{\Delta x}$.

a
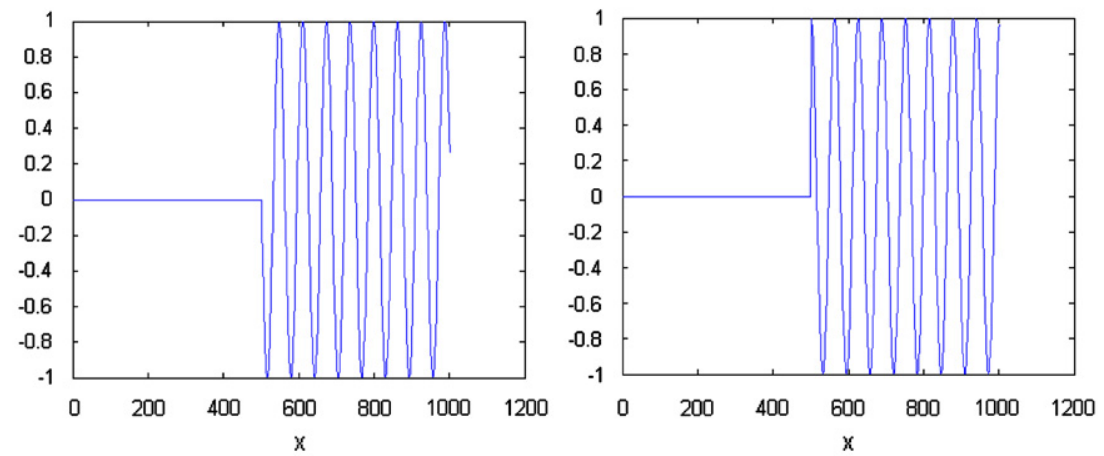

b

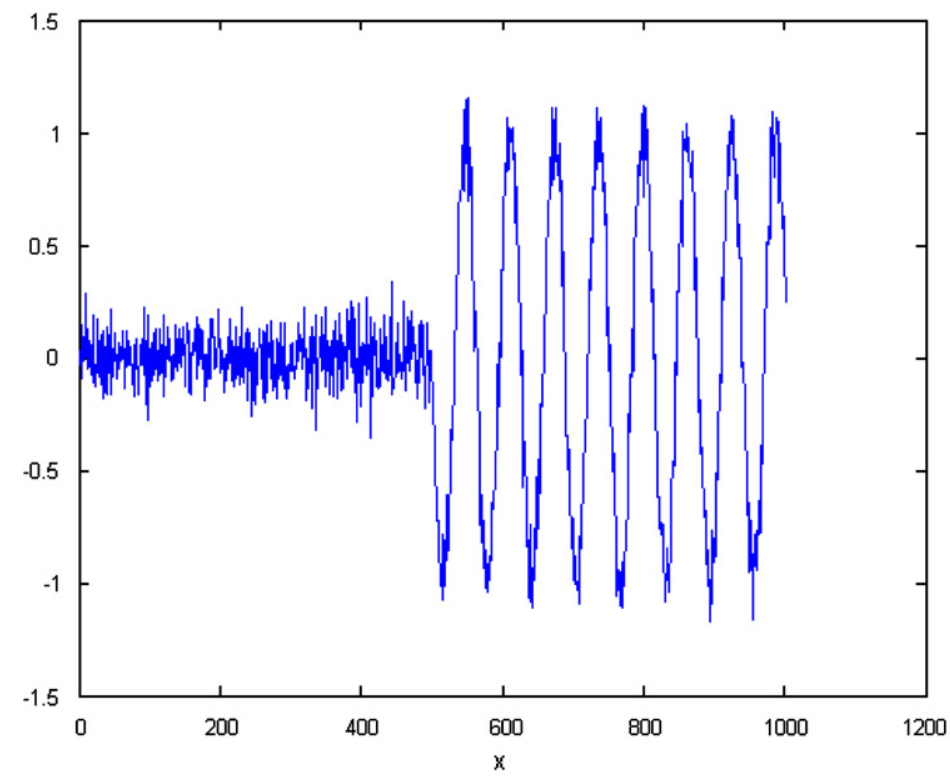

Fig. 3. (a) Noiseless complex band pass signal with discontinuity at $x=500$ (left-real part; right-imaginary part). (b) Noiseless signal of (a) is contaminated with additive Gaussian noise with standard deviation 0.1 ; only real part of signal is shown. (c) Real part of signal smoothed by anisotropic diffusion for band pass signals with $q=4, \Delta t=0.001$, and 4000 iterations. (d) Real part of signal smoothed by traditional anisotropic diffusions with $q=4$, $\Delta t=0.001$, and 4000 iterations. 
C

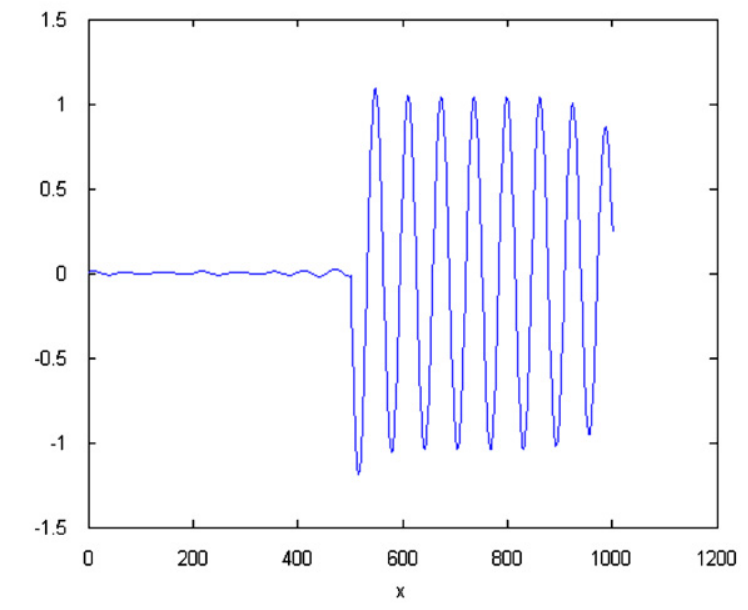

d

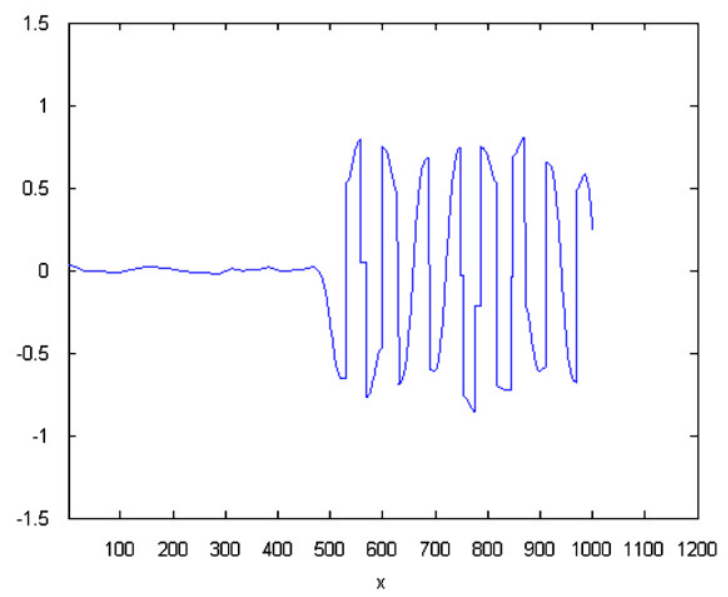

Fig. 3. (Continued)

Function $g(y)$ can be chosen using either Eq. (11) or (12). In this paper, Eq. (11) is used for $g(y)$. Eq. (16) is therefore the main engine of a noise removal algorithm for band pass signals containing discontinuities. The virtual time $t$ therefore corresponds to the iterations in the algorithm. Signal $h$ in Eq. (13) or its discrete implementation of Eq. (16) is complex valued. The Euclidean distance between the smoothed signals in two consecutive iterations is used as a stopping criterion of the algorithm. If this Euclidean distance is less than a user defined threshold $\left(T_{s}\right)$, the algorithm stops.

Let us now discuss the influence of the parameters $T_{s}$, $\Delta t$, and $q$ on the performance of the algorithm. The lower the threshold $T_{s}$, the smoother the signal and the longer the running time of the algorithm.

Parameter $\Delta t$ also plays an important role in this algorithm. Lower values for $\Delta t$ make the algorithm converge to the desired solution slower but with such low values for $\Delta t$, convergence is guaranteed. On the other hand, the algorithm may become unstable, if large values for $\Delta t$ are chosen. In our experiments, $\Delta t$ is always chosen to be less than or equal to 0.001 to guarantee convergence.

Parameter $q$ should be chosen proportional to the amount of change in the discontinuities of the original signal. Large values for $q$ may lead to smoothing discontinuities in the original noiseless signal. On the other hand, if $q$ is small and the signal is too noisy, the smoothed signal may contain false discontinuities associated with noise (and not discontinuities in the original noiseless signal). We also notice that large values for $q$ should be chosen for signals with low SNR (very noisy environment). In a less noisy environment, it is best to choose smaller values for $q$ to avoid smoothing the discontinuities associated with the original noiseless signal. It is also noted that in this paper, we always assume that the original signal contains at least one full cycle of the carrier signal between two consecutive discontinuities to avoid difficulties associated with Heisenberg's uncertainty principle [13].

\section{Numerical results}

Fig. 3(a) shows a noiseless complex band pass signal containing a discontinuity. Gaussian noise is added to this signal to produce the noisy signal shown in Fig. 3(b). The anisotropic diffusion algorithm proposed here is applied to this noisy signal to result in the smoothed signal shown in Fig. 3(c). As can be seen from Fig. 3(c), the discontinuity as well as the high frequency component of the signal is preserved whilst the noise is reduced. Fig. 3(d) depicts the signal smoothed by the traditional anisotropic diffusion (PM) algorithm. As can be seen from Fig. 3(d), the PM algorithm smoothes the noise as well as the high frequency component of the signal (lower amplitude of high frequency oscillations). The traditional anisotropic diffusion also considers the fast rising and falling amplitudes of the high frequency component of the signal as discontinuities and therefore causes serious distortion in the smoothed signal as depicted in Fig. 3(d). The same values for various parameters are used in both algorithms for a fair comparison.

Eq. (16) requires a complex valued signal. Therefore in the example shown in Fig. 3, the signal is complex valued. In practice, however, signals are real valued. In order to be able to use the algorithm proposed here for real valued signals, we propose to employ the Hilbert transform [14] to calculate the imaginary part of the real valued signal to construct a complex valued signal. Fig. 4(a) shows a real valued signal whose Hilbert transform (shown in the same figure) is used as an imaginary part for this real valued signal to compose a complex valued signal. Additive Gaussian noise is added to the real valued signal shown in Fig. 4(a), to produce a noisy signal depicted in Fig. 4(b). The Hilbert transform of this noisy signal shown in Fig. 4(b), is then used as an imaginary part of a complex valued signal. The anisotropic diffusion proposed here for band pass signals is applied to the signal of Fig. 4(b). The real part of the smoothed signal is depicted in Fig. 4(c). Another noiseless band pass signal containing a discontinuity at $x=500$ is shown in Fig. 5(a). This signal is contaminated with additive Gaussian noise 
a

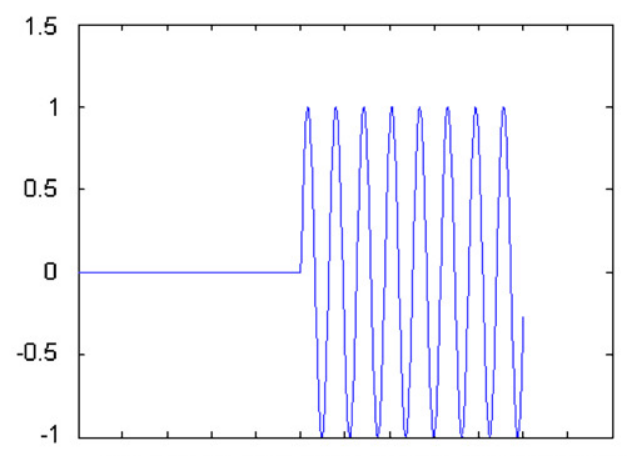

100200300400500600700800900100011001200 $x$

b

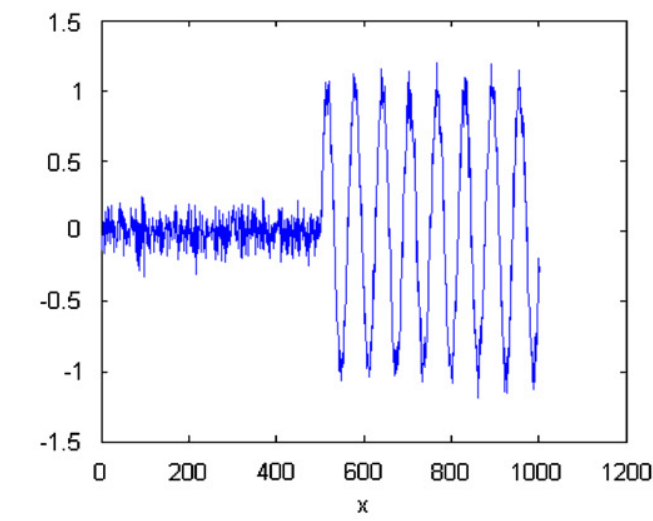

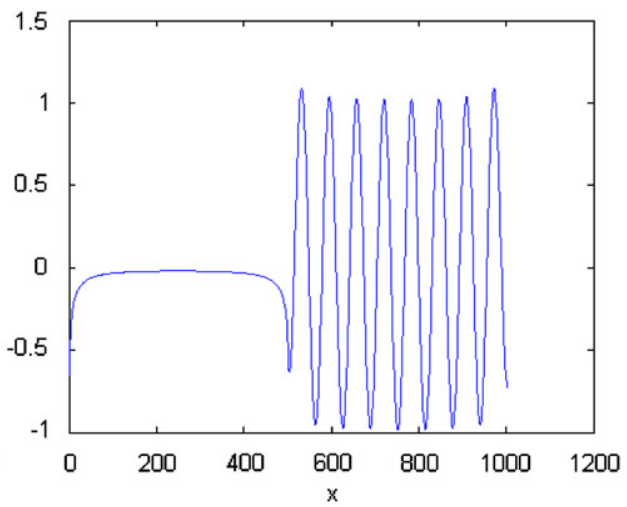

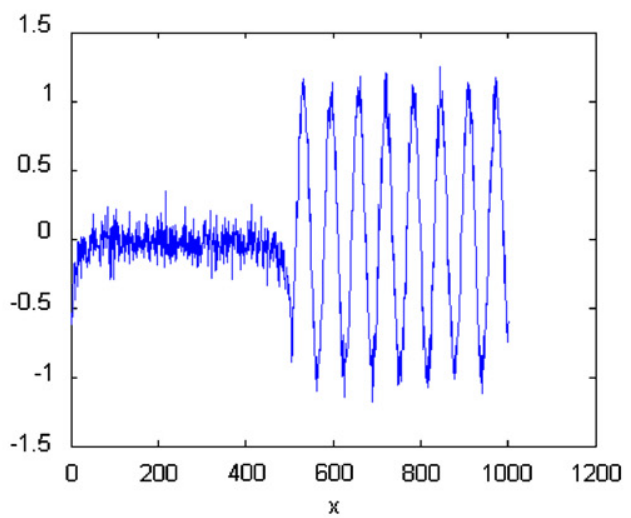

C

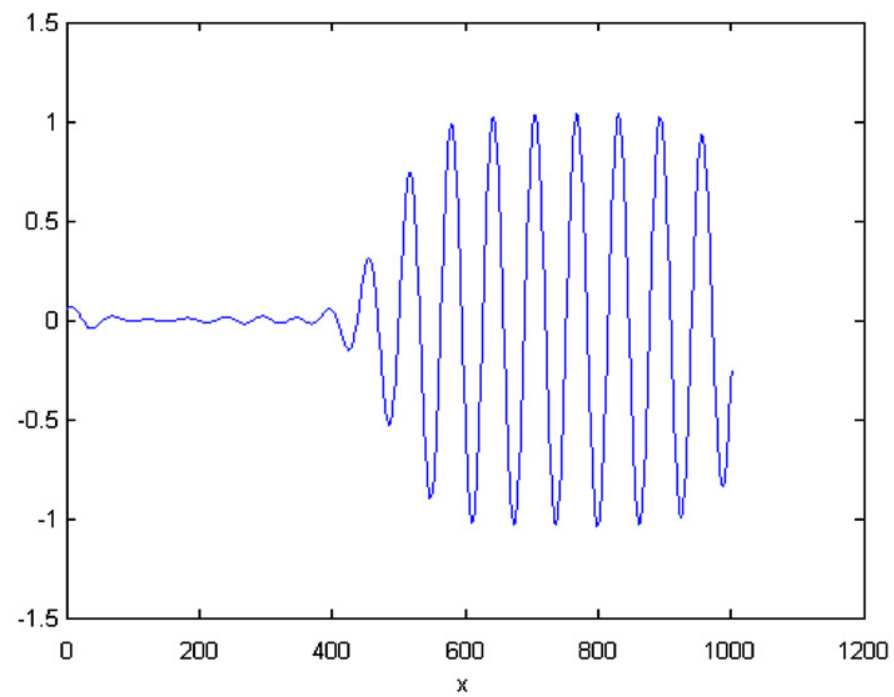

Fig. 4. (a) Real signal (left) and its Hilbert transform (right). (b) Real signal contaminated with additive Gaussian noise with standard deviation 0.1 (left) and its Hilbert transform (right). (c) Real part of smoothed complex valued signal composed in (b) by anisotropic diffusion for band pass signals with $q=4$, $\Delta t=0.001$, and 4000 iterations.

with standard deviation 0.2 to produce the noisy signal shown in Fig. 5(b). The algorithm proposed here is applied to the noisy signal of Fig. 5(b) to obtain the smoothed signal in Fig. 5(c). The traditional anisotropic diffusion is also applied to the noisy signal of Fig. 5(b) to produce the smoothed signal depicted in Fig. 5(d). As can be seen from Fig. 5, the signal smoothed by the algorithm proposed here preserves the discontinuity as well as the high 
a

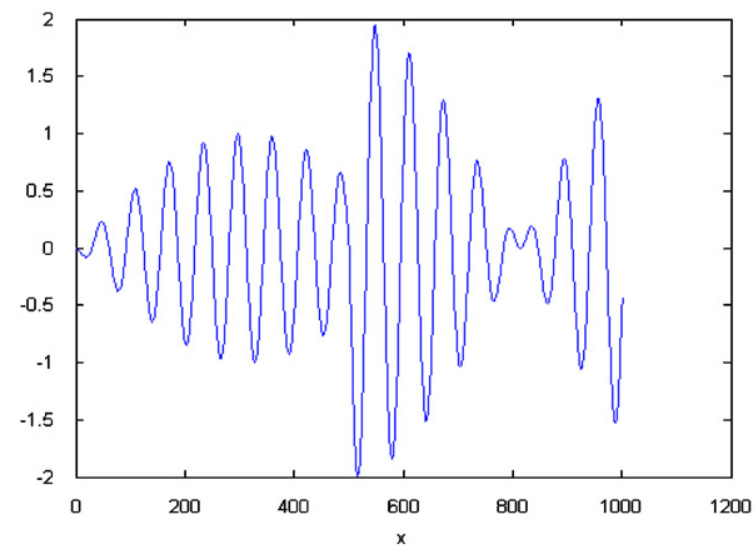

C

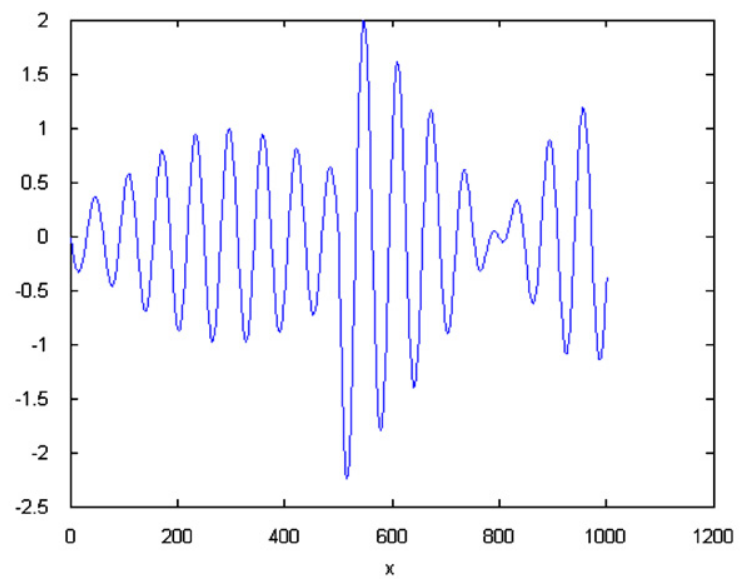

b

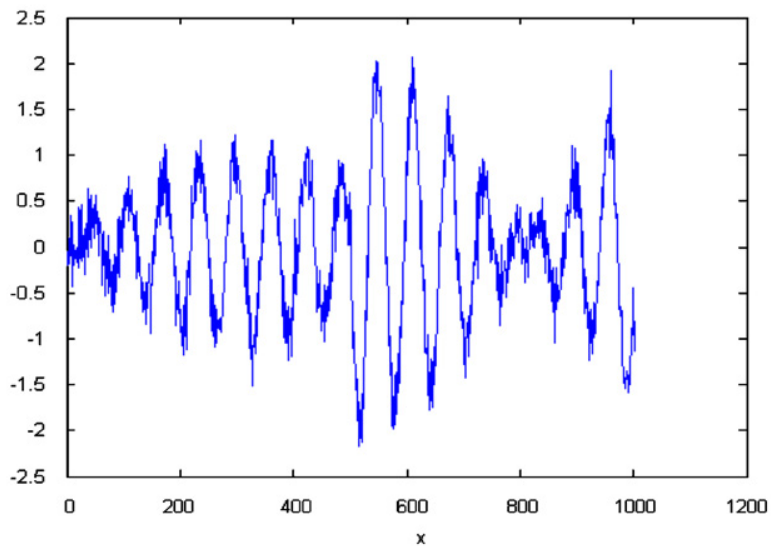

d

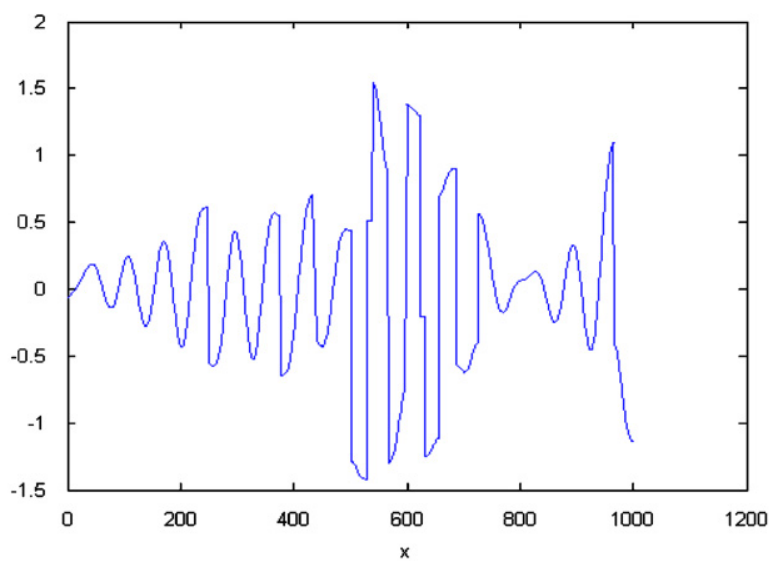

Fig. 5. Superior performance of algorithm proposed here over traditional anisotropic diffusion for a band pass signal whose envelope changes slowly and contains discontinuity. (a) Noiseless band pass signal—envelope of signal varies slowly with respect to $x$ and it has a discontinuity at $x=500$. (b) Additive Gaussian noise with standard deviation 0.2 is added to signal of (a). (c) Smoothed signal obtained by applying algorithm proposed here with $q=6$, $\Delta t=0.001$, and 4000 iterations. (d) Smoothed signal obtained by applying traditional anisotropic diffusion algorithm with $q=6, \Delta t=0.001$, and 4000 iterations.

frequency component (carrier) of the original noiseless signal, whilst it removes the noise. However, the traditional anisotropic diffusion partly smoothes the high frequency component (the signal amplitude is less than that of the original signal) and it also considers the fast falling and rising amplitude of the signal as discontinuity and hence causes serious distortion in the smoothed signal (Fig. 5(d)). The anisotropic diffusion algorithm proposed here is numerically more expensive than the PM algorithm. For example it takes $93.375 \mathrm{~s}$ for a PC with an $800 \mathrm{MHz}$ CPU to run the algorithm proposed here with 4000 iterations as described in Fig. 5 in a Matlab environment (version 6.1). On the other hand, the traditional anisotropic diffusion takes $54.86 \mathrm{~s}$ to run 4000 iterations in the case of the example shown in Fig. 5. Fig. 6(a) depicts a band pass signal with a discontinuity in phase (rather than amplitude). Additive Gaussian noise is added to this signal to produce a noisy signal shown in Fig. 6(b) with $\mathrm{SNR}=0.43$. The anisotropic diffusion algorithm for band pass signals proposed here is applied to this noisy signal to produce the smoothed signal depicted in Fig. 6(c). As can be seen from Fig. 6(c), the algorithm proposed here removes the noise and preserves the discontinuity. The traditional anisotropic diffusion is also applied to the noisy signal of Fig. 6(b) to produce the smoothed signal of Fig. 6(d). As shown in this figure, the traditional algorithm removes the noise but it creates some distortion in the original signal by filtering the high frequency component. The discontinuity is also smoothed by the traditional PM algorithm.

Finally Fig. 7(a) shows a noisy real world signal generated based on the Bell 212A modem standard (DPSK with double-bit symbols) with a $1200 \mathrm{~Hz}$ carrier frequency. This signal represents a 16 bit binary word ("1010111100000101"). The anisotropic diffusion proposed here is applied to this signal to produce the smoothed signal depicted in Fig. 7(b), which preserves the discontinuities and the carrier signal associated with 
a

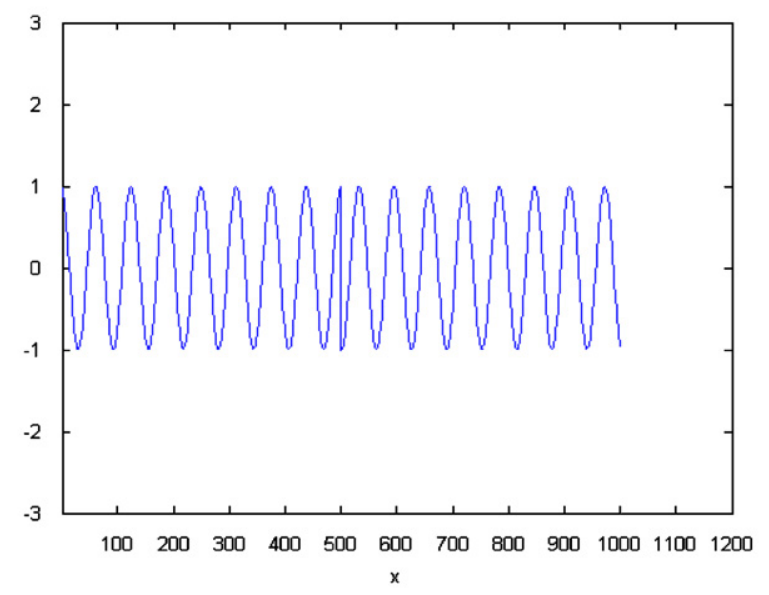

C

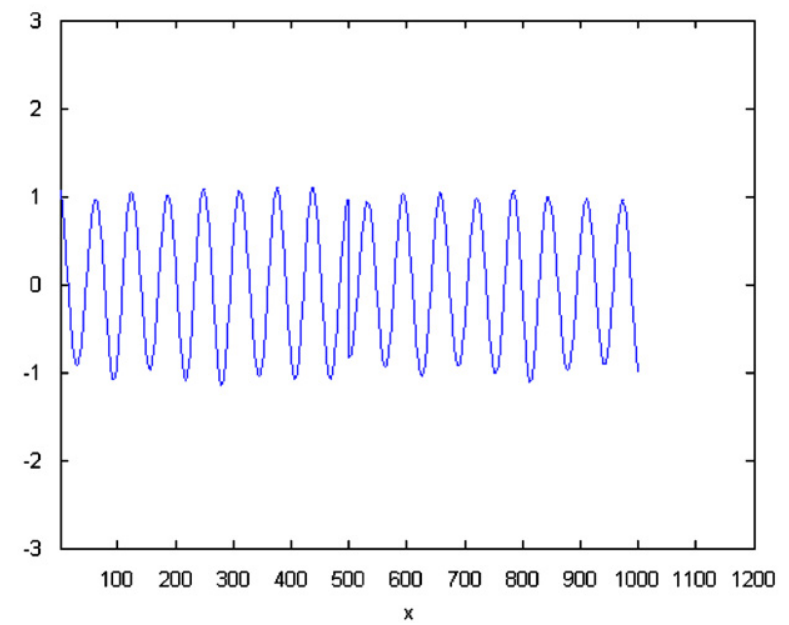

b

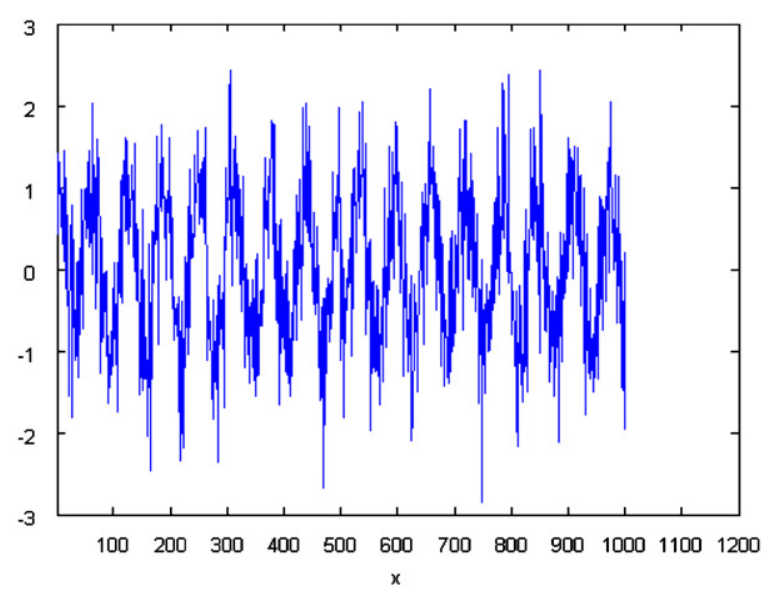

d

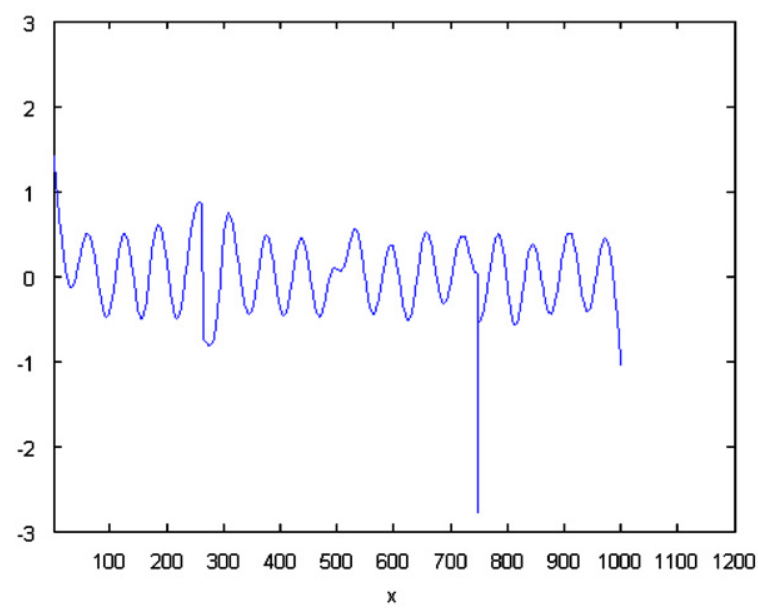

Fig. 6. Superior performance of anisotropic diffusion algorithm proposed in this paper in the presence of excessive noise. (a) Band pass signal with discontinuity in phase at $x=500$. (b) Noisy signal with additive Gaussian noise with standard deviation 0.6 (SNR=0.43). (c) Signal smoothed by algorithm proposed here with $q=15, \Delta t=0.001$, and $T_{s}=0.02$. (d) Signal smoothed by traditional anisotropic diffusion with $q=15, \Delta t=0.001$, and $T_{S}=0.02$.

the original signal. Discontinuities that are preserved by the algorithm proposed here are pointed to by arrows in Fig. 7(b). The traditional anisotropic diffusion also produces the smoothed signal shown in Fig. 7(c). It is noted that the smoothed signal using the traditional anisotropic diffusion has lower amplitude than the original signal due to the low pass smoothing of the PM algorithm. As can be seen from Fig. 7(c), the discontinuities associated with the original signal have also been smoothed or distorted by the traditional anisotropic diffusion. The parameters used in both algorithms in this experiment are $q=15, T_{s}=0.01$, and $\Delta t=0.001$.

\section{Conclusion}

The anisotropic diffusion method proposed by Perona and Malik is extended in this paper for band pass signals containing discontinuities. The mathematical framework for such an algorithm is established here. Such a mathematical framework is best suited for complex valued signals. Therefore Hilbert transform is employed to help use the algorithm proposed here for real valued signals. The method proposed in this paper shows superior performance over the traditional anisotropic diffusion. The method developed here is not unique. Similar results may be obtained by assuming that the relation between low pass and band pass signals are obtained by $h(x, t)=u(x, t) \mathrm{e}^{-i \omega_{0} x}$ rather than Eq. (4). The anisotropic diffusion proposed here for band pass signals is robust in a noisy environment with SNR even less than unity. For the future work, it is interesting to consider signals in which the time interval between consecutive discontinuities is less than a full cycle of the carrier signal. 

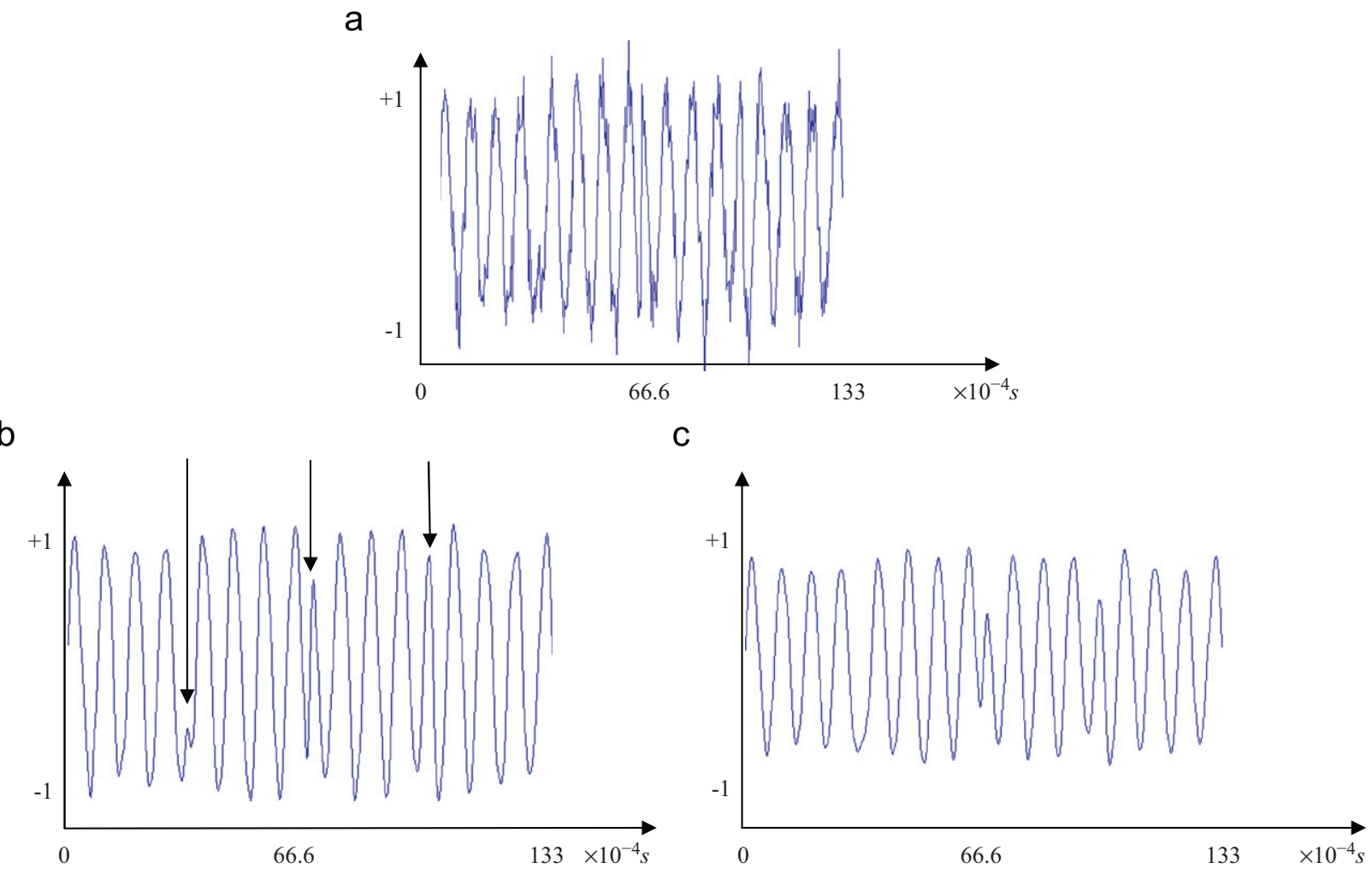

Fig. 7. Noise removal of a real world signal (DPSK with multi-bit symbols) generated using Bell 212A modem standard. (a) Original noisy signal representing a 16 bit binary word 1010111100000101. (b) Signal smoothed by anisotropic diffusion proposed here $\left(q=15, T_{s}=0.01\right.$, and $\Delta t=0.001$; discontinuities of the signal are pointed to by arrows). (c) Signal smoothed by traditional anisotropic diffusion $\left(q=15, T_{s}=0.01\right.$, and $\left.\Delta t=0.001\right)$.

\section{Acknowledgements}

This work was supported in part by the IST program of the European Community, under the PASCAL2 Network of Excellence (IST-2007-216886) and PinView project with Grant no. 216529.

\section{Appendix A. Proof of Theorem 1}

In order to find the propagator of equation (5), we employ Fourier and Laplace transforms with respect to $x$ and $t$, respectively to change differential equation (5) to an algebraic equation, which is easier to solve for $s$. Let us take the Laplace transform with respect to virtual time $t$ from both sides of Eq. (5)

$s H(x, s)-h(x, 0)=K\left(\frac{\partial^{2} H(x, s)}{\partial^{2} x}-2 i \omega_{0} \frac{\partial H(x, s)}{\partial x}-\omega_{0}^{2} H(x, s)\right)$

where $h(x, 0)$ and $H(x, s)$ are the initial condition and the Laplace transform of $h(x, t)$ with respect to $t$, respectively. To derive the propagator of Eq. (5), the initial condition is set to Dirac delta function, i.e. $h(x, 0)=\delta(x)$. By taking the Fourier transform with respect to $x$ from both sides of Eq. (A-1), we arrive at

$s P(\omega, s)-1=K\left(-\omega^{2} P(\omega, s)+2 \omega \omega_{0} P(\omega, s)-\omega_{0}^{2} P(\omega, s)\right)$ where $P(\omega, s)$ is the Fourier transform of $H(x, s)$ with respect to $x . P(\omega, s)$ is solved from Eq. (A-2)

$P(\omega, s)=\frac{1}{\left(s+K\left(\omega-\omega_{0}\right)^{2}\right)}$

By taking the inverse Laplace and Fourier transforms from both sides of Eq. (A-3), the propagator of Eq. (5) is derived as

$P(x, t)=\frac{\exp \left(-\left(x^{2} / 4 K t\right)+i \omega_{0} x\right)}{\sqrt{4 \pi K t}}$

\section{References}

[1] P. Perona, J. Malik, Scale-space and edge detection using anisotropic diffusion, IEEE Transactions on Pattern Recognition and Machine Intelligence 12 (7) (1990) 629-639.

[2] G.W. Wei, Generalized Perona-Malik equation for image restoration, IEEE Signal Processing Letters 6 (7) (1999) 165-167.

[3] F. Catte, P.L. Lions, J.M. Morel, T. Coll, Image selective smoothing and edge detection by nonlinear diffusion, SIAM Journal of Numerical Analysis 29 (1) (1992) 182-193.

[4] M. Kichenassamy, The Perona-Malik paradox, SIAM Journal on Applied Mathematics 57 (5) (1997) 1328-1342.

[5] M. Nitzberg, T. Shiota, Nonlinear image filtering with edge and corner enhancement, IEEE Transactions on Pattern Analysis and Machine Intelligence 14 (8) (1992) 826-833.

[6] R.T. Whitaker, S.M. Pizer, A multi-scale approach to non-uniform diffusion, GVGIP: Image Understanding 57 (1) (1993) 99-110.

[7] Y.L. You, W. Xu, A. Tannenbaum, M. Kaveh, Behaviour analysis of anisotropic diffusion in image processing, IEEE Transactions on Image Processing 5 (11) (1996) 1539-1553. 
[8] G. Gerig, O. Kubler, R. Kikinis, F. Jolesz, Nonlinear anisotropic filtering of MRI data, IEEE Transactions on Medical Imaging 11 (2) (1992) 221-232.

[9] G. Sapiro, D.L. Ringach, Anisotropic diffusion of multi-valued images with applications to color filtering, IEEE Transactions on Image Processing 5 (11) (1996) 1582-1586.

[10] M.J. Black, G. Sapiro, D.H. Marimont, D. Heeger, Robust anisotropic diffusion, IEEE Transactions on Image Processing 7 (3) (1998) 421-432.
[11] D. Tschumperle, Fast anisotropic smoothing of multi-valued images using curvature-preserving PDEs, International Journal of Computer Vision 68 (1) (2006) 65-82.

[12] H. Luo, L. Zhu, H. Ding, Coupled anisotropic diffusion for image selective smoothing, Signal Processing 86 (7) (2006) 1728-1736.

[13] V. Harvin, B. Joricke, The Uncertainty Principle in Harmonic Analysis, Springer-Verlag, 1994.

[14] A.V. Oppenheim, R.W. Schafer, Digital Signal Processing, PrenticeHall International Inc., 1975. 